\title{
BMJ Open Pneumonia diagnosis in childhood and incidence of leukaemia, lymphoma and brain cancer: a Danish nationwide cohort study
}

Kirstine Kobberøe Søgaard, ${ }^{1,2}$ Dóra Körmendiné Farkas, ${ }^{1}$ Henrik Toft Sørensen ${ }^{1}$

To cite: Søgaard KK, Farkas DK, Sørensen HT. Pneumonia diagnosis in childhood and incidence of leukaemia, lymphoma and brain cancer: a Danish nationwide cohort study. BMJ Open 2017;7:e019860. doi:10.1136/ bmjopen-2017-019860

- Prepublication history and additional material for this paper are available online. To view these files, please visit the journal online (http://dx.doi org/10.1136/bmjopen-2017019860).

Received 2 0ctober 2017 Revised 16 November 2017 Accepted 1 December 2017
CrossMark

${ }^{1}$ Department of Clinical Epidemiology, Aarhus University Hospital, Aarhus, Denmark ${ }^{2}$ Department of Clinical Microbiology, Aalborg University Hospital, Aalborg, Denmark

Correspondence to Dr Kirstine Kobberøe Søgaard; kks@clin.au.dk

\section{ABSTRACT}

Objectives There is an ongoing debate on the possible association between infections in early childhood and subsequent cancer risk, but it remains unclear if a hospital admission for infection is associated with risk of childhood cancer diagnosis. We examined if a hospitalbased diagnosis of pneumonia was a clinical marker of the three most common childhood cancers.

Design Population-based cohort study.

Setting Denmark, hospital diagnoses, 1994-2013.

Methods Using national health registries, we compared the observed incidence of leukaemia, lymphoma and brain cancer among 83935 children with a hospitalbased pneumonia diagnosis with that expected among children in the general population. We calculated absolute cancer risks and standardised incidence ratios (SIRs) as a measure of relative risk.

Results The cancer SIRs were substantially increased during the first 6 months of follow-up; lymphoid leukaemia: 6.2 (95\% Cl 3.5 to 10.3); myeloid leukaemia: 14.8 (95\% Cl 6.0 to 30.6); Hodgkin's lymphoma: 60.8 (95\% Cl 26.2 to 120), non-Hodgkin's lymphoma: 15.9 (95\% Cl 5.2 to 37.2$)$ and brain cancer: $4.4(95 \% \mathrm{Cl}$ 1.9 to 8.7). The 6 -month absolute risks of leukaemia, lymphoma and brain cancer were all low, reaching $0.05 \%$ when combined. An increased risk persisted beyond 5 years for non-Hodgkin's lymphoma and brain cancer. However, the 5-year absolute cancer risk was $0.14 \%$.

Conclusions The short-term incidence of leukaemia, lymphoma and brain cancer was higher than expected and persisted beyond 5 years for non-Hodgkin's lymphoma and brain cancer. However, the absolute cancer risk was low.

\section{INTRODUCTION}

Presenting signs of cancer in children may be vague and overlap with those of common childhood conditions. While the disease course for leukaemia is often short, the symptoms of brain cancer may present already 2 years before diagnosis. General practitioners (GPs) are often essential in the early diagnostic pathways, ${ }^{2}$ but it is plausible that cancer in some children debut with acute clinical disease
Strengths and limitations of this study

- We performed a population-based study using a well-established method to calculate cancer risk, estimating both absolute and relative risks.

- We focused on hospital-based pneumonia diagnosis rather than a composite of infections.

- We could not separate the order of diagnoses among those diagnosed with pneumonia and cancer during same admission.

- We did not have information from the medical files and therefore could not depict if some children were initially misdiagnosed as pneumonia and later diagnosed with mediastinal lymphoma.

necessitating hospitalisation. Hospital admission for infection is not clearly associated with risk of childhood cancer. Some studies have shown that overall there is no association with previous admission for infectious diseases and risk of leukaemia, ${ }^{3}$ while others have found that children with common infections requiring hospitalisation potentially have a $50 \%$ higher risk of a subsequent leukaemia diagnosis. ${ }^{4}$ Specifically, a hospital diagnosis of pneumonia may be associated with subsequent increased risk of a leukaemia diagnosis. ${ }^{5}$ Pneumonia is a frequent cause of community-acquired infection leading to hospital contact (annual incidence is up to 40 per $10000)^{6}$; therefore, any association with cancer could be clinically relevant. If absolute cancer risks in children with a hospital-based diagnosis of pneumonia are high, then this could have implications for the diagnostic approach in these children.

We assessed absolute and relative risks of the three most common childhood cancers, leukaemia, lymphoma and brain cancer $^{7}$ subsequent to a first hospital-based diagnosis of pneumonia. 
Table 1 Risk of leukaemia, lymphoma and brain cancer, stratified analysis

\begin{tabular}{|c|c|c|c|}
\hline & $\mathbf{n}$ & O/E & SIR $(95 \% \mathrm{CI})$ \\
\hline All & 83935 & $168 / 90$ & 1.9 (1.6 to 2.2$)$ \\
\hline Boys & 47650 & $89 / 53$ & 1.7 (1.3 to 2.1$)$ \\
\hline Girls & 36285 & $79 / 37$ & 2.2 (1.7 to 2.7$)$ \\
\hline \multicolumn{4}{|l|}{ Age } \\
\hline $0-4$ & 70476 & $135 / 76$ & 1.8 (1.5 to 2.1$)$ \\
\hline $5-9$ & 8145 & $14 / 8$ & 1.7 (0.9 to 2.8$)$ \\
\hline $10-14$ & 3114 & $8 / 3$ & 2.3 (1.0 to 4.5$)$ \\
\hline $15-17$ & 2200 & $11 / 3$ & $4.2(2.1$ to 7.5$)$ \\
\hline \multicolumn{4}{|c|}{ Previous hospitalisations } \\
\hline 0 & 18114 & $20 / 17$ & $1.2(0.7$ to 1.9$)$ \\
\hline 1 & 45161 & $94 / 50$ & 1.9 (1.5 to 2.3$)$ \\
\hline 2 & 11953 & $24 / 14$ & 1.8 (1.1 to 2.6$)$ \\
\hline $3+$ & 8707 & $30 / 10$ & 3.1 (2.1 to 4.4$)$ \\
\hline \multicolumn{4}{|l|}{ Calendar period } \\
\hline 1994-1998 & 21618 & $96 / 81$ & $1.2(1.0$ to 1.4$)$ \\
\hline 1999-2003 & 21067 & $61 / 49$ & 1.3 (1.0 to 1.6$)$ \\
\hline 2004-2008 & 20644 & $54 / 29$ & 1.9 (1.4 to 2.5$)$ \\
\hline 2009-2013 & 20607 & $30 / 12$ & 2.5 (1.7 to 3.5$)$ \\
\hline \multicolumn{4}{|c|}{ Congenital malformations } \\
\hline Yes & 8733 & $31^{\star} / 9$ & 3.7 (2.5 to 5.2$)$ \\
\hline Down syndrome & 489 & $9 / 1$ & $16.2(7.4$ to 30.8$)$ \\
\hline No & 75202 & $137 / 81$ & 1.7 (1.4 to 2.0$)$ \\
\hline \multicolumn{4}{|l|}{ Immune deficiency } \\
\hline Yes & 137 & $2 / 0.1$ & 17.5 (2.1 to 63.2$)$ \\
\hline No & 83798 & $166 / 90$ & 1.9 (1.6 to 2.2$)$ \\
\hline \multicolumn{4}{|c|}{ Imaging examination (after 2002) } \\
\hline Yes & 36230 & $66 / 25$ & 2.7 (2.1 to 3.4$)$ \\
\hline No & 13280 & $9 / 8$ & $1.1(0.5$ to 2.1$)$ \\
\hline
\end{tabular}

Congenital malformations included conditions related to the nervous system (Q00-Q07); eye, ear, face and neck (Q10-Q18); the circulatory system (Q20-Q28); the respiratory system (Q30-Q34); cleft lip and cleft palate (Q35-Q37); the digestive system (Q38-Q45); genital organs (Q50-Q56); the urinary system (Q60-Q64); malformations and deformations of the musculoskeletal system (Q65-Q79) and other (Q80-Q99).

$\mathrm{O} / \mathrm{E}$, observed/expected number of cancer; SIR, standardised incidence ratio.

\section{MATERIALS AND METHODS}

The source population for this registry-based cohort was all Danish children aged 0-17 years who were alive between 1994 and 2013 ( $\mathrm{n}=2884$ 552). Access to medical care including hospital admissions is free of charge (tax paid). The Danish National Patient Registry (DNPR) captures all contacts with Danish hospitals, ${ }^{8}$ and records discharge diagnosis using the International Classification of Diseases (currently the 10th revision). The Danish Cancer Registry (DCR) records incident cancers in Denmark using ICD-10 and ICD-0-3 morphology codes. ${ }^{9}$
We identified all children with a first-time hospital-based (inpatient, outpatient clinic and emergency room) diagnosis of pneumonia recorded in the DNPR during 1994-2013. We linked these cases to the DCR (using a unique personal identification) to identify incident cancers, and then to exclude children with previous cancer diagnoses. Owing to the low cancer incidence in the cohort, we had sufficient sample size to estimate only the risks of the three most common childhood cancers. We used information on registrations of chest imaging during hospitalisation (recorded since 2002) to examine the proportion of imaging-confirmed pneumonia diagnoses. We obtained information from the DNPR on prevalent diagnoses of immune deficiencies and congenital diseases, including Down syndrome. We searched for hospitalisations before pneumonia diagnosis to elucidate the extent of previous hospital contacts. All codes used in the study are provided in the online supplementary appendix.

\section{Statistical analysis}

The children were followed for the occurrence of cancer from the date of the pneumonia diagnosis until death, emigration or end of follow-up 30 November 2013. We computed the absolute cancer risk at 6 months and 5 years following the pneumonia diagnosis.

We compared the observed cancer incidence among children with pneumonia with that expected among children in the general population (based on national cancer incidence rates by age, sex and calendar year). Standardised incidence ratios (SIRs) were calculated as a measure of relative risk. We computed SIRs for acute myeloid leukaemia, acute lymphoid leukaemia, Hodgkin lymphoma, non-Hodgkin's lymphoma and brain cancer. Follow-up was divided into first 6 months, 6 months to 5 years and $5+$ years. We stratified the patients by gender, age, calendar period and prevalent disease to examine its potential impact on cancer risk.

All statistical analyses were conducted using the SAS statistical software package V.9.2 (SAS Institute).

\section{RESULTS}

\section{Patient characteristics}

We followed 83935 children with pneumonia and no previous cancer diagnosis for a median of 10 years. The incidence of pneumonia diagnosis was stable over the 20 -year period. In the cohort as a whole, $47650(57 \%)$ were boys, and median age was 1.5 years. Pneumonia diagnosis was made during an inpatient stay among $89 \%$, during an outpatient clinic visit in $6 \%$ and in the emergency room in $5 \%$ of the children. Pneumonia was registered as the main condition leading to hospitalisation for 69479 (83\%) children. Of the 49510 children diagnosed with pneumonia after 2002, 36230 (73\%) had chest imaging performed during their hospital contact. Pneumonia was registered as caused by bacteria in $14 \%$ and by viruses in $22 \%$ of children in the study 
Table 2 Risk of leukaemia, lymphoma and brain cancer by follow-up interval

\begin{tabular}{|c|c|c|c|c|c|c|}
\hline \multirow[b]{3}{*}{ Lymphoid leukaemia* } & \multicolumn{6}{|c|}{ Observed/expected numbers of cancer and SIRs $(95 \% \mathrm{Cl})$} \\
\hline & \multicolumn{2}{|c|}{$0-<6$ months of follow-up } & \multicolumn{2}{|c|}{6 months $-<5$ years } & \multicolumn{2}{|c|}{$5+$ years } \\
\hline & $15 / 2$ & 6.2 (3.5 to 10.3$)$ & $27 / 16$ & $1.7(1.1$ to 2.4$)$ & $8 / 10$ & $0.8(0.3$ to 1.5$)$ \\
\hline Hodgkin's lymphomał & $8 /<1$ & $60.8(26.2$ to 120$)$ & $2 / 1$ & $1.4(0.2$ to 5.0$)$ & $6 / 8$ & $0.7(0.3$ to 1.5$)$ \\
\hline Non-Hodgkin's lymphoma§ & $5 /<1$ & 15.9 (5.2 to 37.2$)$ & $8 / 3$ & 2.7 (1.2 to 5.3$)$ & $11 / 6$ & 1.7 (0.9 to 3.0$)$ \\
\hline
\end{tabular}

Some of the most frequent subtypes of cancer were as follows:

*42 of 50 children had precursor cell lymphoblastic leukaemia, not otherwise specified.

†10 of 17 children had acute myeloid leukaemia, not otherwise specified.

$\$ 10$ of 16 children had nodular sclerosis classical Hodgkin's lymphoma.

$\S 5$ of 24 children had diffuse large B cell, not otherwise specified; 5 had mature T-cell lymphoma, not otherwise specified; 4 had Burkitt lymphoma, not otherwise specified and 4 had anaplastic large cell lymphoma.

I15 of 61 children had glioblastoma; 8 had medulloblastoma, not otherwise specified, and 28 had other or unspecified brain cancer.

SIR, standardised incidence ratio.

cohort (see the online supplementary appendix for specification of aetiology), while the agent was unspecified for $64 \%$ of the children. Among the children, 8733 (10\%) had a congenital malformation (table 1), including 489 children with Down syndrome. The prevalence of immune deficiencies recorded in the DNPR at the time of pneumonia diagnosis was low $(\mathrm{n}=137)$. The majority had one or no previous hospitalisations, while $25 \%$ had two or more previous hospitalisations with a minimum duration of 3 days.

\section{Risk of leukaemia, lymphoma and brain cancer}

A total of 168 cancers were diagnosed during follow-up (826 281 person-years), including 37 diagnosed with cancer within 1 month, adding up to 43 cancers within the first 6 months following the pneumonia episode. The most frequent subtypes of cancer are provided in table 2 .

The absolute risks of leukaemia, lymphoma and brain cancer were all low, combined it reached $0.05 \%$ at 6 months and $0.14 \%$ at 5 years.

During complete follow-up, the combined SIR of leukaemia, lymphoma and brain cancer was increased by almost twofold (table 1). The SIRs were substantially increased during the first 6 months of follow-up: 6.2 (95\% CI 3.5 to 10.3) for lymphoid leukaemia, 14.8 (95\% CI 6.0 to 30.6) for myeloid leukaemia, 60.8 (95\% CI 26.2 to 120) for Hodgkin's lymphoma, 15.9 (95\% CI 5.2 to 37.2 ) for non-Hodgkin's lymphoma and 4.4 (95\% CI 1.9 to 8.7) for brain cancer (table 2). The increased risk of lymphoid leukaemia, myeloid leukaemia and non-Hodgkin's lymphoma persisted up to 5 years after a hospital pneumonia diagnosis. Beyond 5 years of follow-up, more children than expected were diagnosed with non-Hodgkin's lymphomas and brain cancers (table 2).

The stratified analyses for the overall cancer occurrence showed slightly higher SIRs among girls than among boys. Children up to 14 years of age had an approximately twofold increased SIR, whereas teenagers aged 15-17 years had a fourfold increased SIR (table 1). The SIR increased over time from 1.2-fold increased during 1994-1998 to 2.5-fold increased during 2009-2013. Children with imaging-confirmed diagnoses had a SIR of almost three, whereas children without confirmed diagnosis had a SIR around the unity (table 1 ). Though numbers were low, children diagnosed with immune deficiencies or congenital malformations had higher SIRs for cancer than children without these conditions. However, importantly the increased risk was not confined to children with such known conditions. While the overall SIR for children without previous hospitalisations was around unity, the SIR for children with one or two visits was almost twofold increased, and the SIR for those with three or more visits was threefold increased (table 1).

\section{DISCUSSION}

We found that a hospital-based diagnosis of pneumonia was a rare presentation of occult childhood cancer. The children hospitalised with pneumonia had a higher short-term incidence of leukaemia, lymphoma and brain cancer than expected and had a persistently increased risk of non-Hodgkin's lymphoma and brain cancer more than 5 years after the pneumonia. However, the absolute risk of cancer was low, which accords with the low incidence of childhood malignancies.

The association between hospital-based diagnoses of common infections and subsequent cancer occurrence are conflicting. A Danish population-based study found no overall association with previous admission for infectious diseases and risk of leukaemia. However, the study did not explore risks according to type of infection. ${ }^{3}$ By contrast, a French case-control study indicated that common infections occurring in children during the first year of life potentially were associated with decreased risk of leukaemia, whereas children with infections requiring 
hospitalisation could be at increased risk of a leukaemia diagnosis. ${ }^{4}$ Supporting the latter finding, a Taiwan casecontrol study found that a hospital diagnosis of pneumonia was associated with subsequent increased risk of myeloid leukaemia diagnosis. ${ }^{5}$ We found that children presenting with pneumonia had a subsequent higher occurrence of leukaemia, lymphoma and brain cancer than other Danish children.

The strengths of our study include its population-based design in the setting of a uniformly organised healthcare system. In agreement with the overall completeness of chest X-ray records in the DNPR, ${ }^{10}$ we found that the majority of diagnoses were based on imaging procedures. In addition, cancer diagnoses in the DCR have high completeness and validity. ${ }^{9}$ We included children with immune deficiency and congenital malformations, which are known to have higher incidence of both pneumonia and cancer. However, in the analyses excluding these children, the results remained unchanged. Our study also had potential limitations. We could not separate the order of the diagnoses among those diagnosed with pneumonia and cancer during same admission nor identify children in whom lymphoma was initially falsely interpreted as pneumonia. We used hospital-based diagnosis of pneumonia within the setting of a developed country. Accordingly, generalisability may be transferable to other industrial Western societies but not necessarily to undeveloped countries neither to pneumonia diagnosis in the general practice setting.

There are several possible explanations for our findings. Chest X-rays and blood tests performed as part of workup for pneumonia may have yielded findings suspicious of cancer leading on to further examinations. In addition, lymphoma with a mediastinal mass or with lung involvement may have initially been misdiagnosed as pneumonia in some cases. Children with cancer have higher GP consultation rates than controls in the year preceding the cancer diagnosis. ${ }^{11}$ In agreement, we found higher SIRs for children with previous hospital contacts, thus pneumonia may not have been the first clinical disease in all children. Although increased diagnostic effort may partially explain the higher short-term occurrence of cancer, it is unlikely to explain the increased risk of lymphoma and brain cancer observed more than 5 years after pneumonia. There is an ongoing debate on whether or not infectious diseases in children may modulate the child's immune response potentially leading to decreased or increased risk of cancer. ${ }^{12-14}$ We did not attempt to address or clarify this hypothesis as our data would not allow us to do so. However, we speculate if the associations demonstrated may be due to cancer-related impairment of the immune system, making a child more vulnerable to severe infections such as pneumonia. In children with an aggressive type of cancer, the infection may lead on to cancer diagnosis, whereas in children with a more indolent cancer, the infection occurs in the preclinical phase. ${ }^{1}$
Based on the low absolute cancer risks observed, our findings do not warrant a change in the workup of children diagnosed with pneumonia during a hospital admission.

Contributors HTS and KKS conceived the study idea. KKS reviewed the literature. KKS and HTS designed the study and directed the analyses, which were carried out by DKF. All authors participated in the interpretation of the results. KKS organised the writing and wrote the initial drafts. All authors critically revised the manuscript for intellectual content and approved the final version.

Funding This work was supported by the Danish Childhood Cancer Foundation and by the Program for Clinical Research Infrastructure (PROCRIN) established by the Lundbeck Foundation and the Novo Nordisk Foundation.

Competing interests None declared.

Ethics approval The study was approved by the Danish Data Protection Agency, record number 1-16-02-1-08.

Provenance and peer review Not commissioned; externally peer reviewed.

Data sharing statement № additional data are available.

Open Access This is an Open Access article distributed in accordance with the Creative Commons Attribution Non Commercial (CC BY-NC 4.0) license, which permits others to distribute, remix, adapt, build upon this work non-commercially, and license their derivative works on different terms, provided the original work is properly cited and the use is non-commercial. See: http://creativecommons.org/ licenses/by-nc/4.0/

(c) Article author(s) (or their employer(s) unless otherwise stated in the text of the article) 2017. All rights reserved. No commercial use is permitted unless otherwise expressly granted.

\section{REFERENCES}

1. Klitbo DM, Nielsen R, Illum NO, et al. Symptoms and time to diagnosis in children with brain tumours. Dan Med Bull 2011;58:A4285.

2. Ahrensberg JM, Hansen RP, Olesen F, et al. Presenting symptoms of children with cancer: a primary-care population-based study. $\mathrm{Br} \mathrm{J}$ Gen Pract 2012;62:458-65.

3. Vestergaard TR, Rostgaard K, Grau K, et al. Hospitalisation for infection prior to diagnosis of acute lymphoblastic leukaemia in children. Pediatr Blood Cancer 2013;60:428-32.

4. Ajrouche R, Rudant J, Orsi L, et al. Childhood acute lymphoblastic leukaemia and indicators of early immune stimulation: the Estelle study (SFCE). Br J Cancer 2015;112:1017-26.

5. Chang JS, Tsai CR, Tsai YW, et al. Medically diagnosed infections and risk of childhood leukaemia: a population-based case-control study. Int J Epidemiol 2012;41:1050-9.

6. Ostapchuk M, Roberts DM, Haddy R. Community-acquired pneumonia in infants and children. Am Fam Physician 2004;70:899-908.

7. Country Summary. Cancer research UK. 2010. UK: cancer incidence, 2013.

8. Schmidt M, Schmidt SA, Sandegaard JL, et al. The Danish National Patient Registry: a review of content, data quality, and research potential. Clin Epidemiol 2015;7:449-90.

9. Storm HH, Michelsen EV, Clemmensen IH, et al. The Danish Cancer Registry: history, content, quality and use. Dan Med Bull 1997;44:535-9.

10. Hjertholm P, Flarup KR, Guldbrandt LM, et al. The completeness of chest X-ray procedure codes in the Danish National Patient Registry. Clin Epidemiol 2017;9:151-6.

11. Ahrensberg JM, Fenger-Grøn M, Vedsted P. Use of primary care during the year before childhood cancer diagnosis: a nationwide population-based matched comparative study. PLoS One 2013;8:e59098.

12. Greaves M. Infection, immune responses and the aetiology of childhood leukaemia. Nat Rev Cancer 2006;6:193-203.

13. Cardwell CR, McKinney PA, Patterson CC, et al. Infections in early life and childhood leukaemia risk: a UK case-control study of general practitioner records. Br J Cancer 2008;99:1529-33.

14. Kim AS, Eastmond DA, Preston RJ. Childhood acute lymphocytic leukemia and perspectives on risk assessment of early-life stage exposures. Mutat Res 2006;613:138-60. 